\title{
Chronic lymphocytic leukemia requires BCL2 to sequester prodeath BIM, explaining sensitivity to BCL2 antagonist ABT-737
}

\author{
Victoria Del Gaizo Moore, ${ }^{1}$ Jennifer R. Brown, ${ }^{1}$ Michael Certo, ${ }^{1}$ \\ Tara M. Love, ${ }^{2}$ Carl D. Novina, ${ }^{2}$ and Anthony Letai ${ }^{1}$
}

${ }^{1}$ Department of Medical Oncology and 2Department of Cancer Immunology and AIDS, Dana-Farber Cancer Institute, Boston, Massachusetts, USA.

\begin{abstract}
Antiapoptotic B cell leukemia/lymphoma 2 (BCL2) family proteins are expressed in many cancers, but the circumstances under which these proteins are necessary for tumor maintenance are poorly understood. We exploited a novel functional assay that uses BCL2 homology domain 3 (BH3) peptides to predict dependence on antiapoptotic proteins, a strategy we call $\mathrm{BH} 3$ profiling. $\mathrm{BH} 3$ profiling accurately predicts sensitivity to BCL2 antagonist ABT-737 in primary chronic lymphocytic leukemia (CLL) cells. BH3 profiling also accurately distinguishes myeloid cell leukemia sequence 1 (MCL1) from BCL2 dependence in myeloma cell lines. We show that the special sensitivity of CLL cells to BCL2 antagonism arises from the requirement that BCL2 tonically sequester proapoptotic BIM in CLL. ABT-737 displaced BIM from BCL2's BH3-binding pocket, allowing BIM to activate BAX, induce mitochondrial permeabilization, and rapidly commit the CLL cell to death. Our experiments demonstrate that BCL2 expression alone does not dictate sensitivity to ABT-737. Instead, BCL2 complexed to BIM is the critical target for ABT-737 in CLL. An important implication is that in cancer, BCL2 may not effectively buffer chemotherapy death signals if it is already sequestering proapoptotic BH3-only proteins. Indeed, activator BH3-only occupation of BCL2 may prime cancer cells for death, offering a potential explanation for the marked chemosensitivity of certain cancers that express abundant BCL2, such as CLL and follicular lymphoma.
\end{abstract}

\section{Introduction}

Proteins in the B cell leukemia/lymphoma 2 (BCL2) family are key arbiters of the commitment to programmed cell death at the mitochondrion $(1,2)$. This family can be divided into 3 groups: the antiapoptotic proteins, including BCL2, BCL- $\mathrm{X}_{\mathrm{L}}$, and myeloid cell leukemia sequence 1 (MCL1); the multidomain (BH1, $\mathrm{BH} 2$, and $\mathrm{BH} 3$-containing; $\mathrm{BH}, \mathrm{BCL} 2$ homology domain) proapoptotic proteins $\mathrm{BAX}$ and $\mathrm{BAK}$; and the prodeath $\mathrm{BH} 3$-only proteins, including $\mathrm{BIM}, \mathrm{BID}, \mathrm{BAD}, \mathrm{BIK}$, and NOXA. In response to a wide range of death and derangement signals, $\mathrm{BH} 3$-only members are activated $(3,4)$. To exert their prodeath function, they require an intact $\mathrm{BH} 3$ domain (5) as well as the presence of $\operatorname{BAX}$ or $\operatorname{BAK}(6,7)$. Certain $\mathrm{BH} 3$ domains, such as BID and BIM, induce allosteric changes and oligomerization of BAX and BAK and are termed activators $(8,9)$. Mitochondrial outer membrane permeabilization (MOMP) follows $\mathrm{BAX}$ and BAK oligomerization, allowing release of cytochrome $c$ and other proapoptotic factors into the cytosol $(10,11)$. Cytochrome $c$ then interacts with caspase- 9 and apoptosis protease-activating factor-1 (APAF-1) to form a holoenzyme that proteolytically activates effector caspase-3, causing widespread proteolysis and cell death.

BCL2 and other antiapoptotic proteins can inhibit apoptosis by sequestering activator $\mathrm{BH} 3$-only molecules, preventing oligomerization of BAX and BAK $(8,12,13)$. A class of BH3-only BH3

Nonstandard abbreviations used: BCL2, B cell leukemia/lymphoma 2; BH, BCL2 homology domain; CLL, chronic lymphocytic leukemia; FL, follicular lymphoma; MCL1, myeloid cell leukemia sequence 1; MOMP, mitochondrial outer membrane permeabilization; shRNA, short hairpin RNA

Conflict of interest: A. Letai is a consultant for Abbott Laboratories. A tool used in these studies, $\mathrm{BH} 3$ profiling, is the subject of a US patent application submitted by Dana-Farber Cancer Institute.

Citation for this article: J. Clin. Invest. 117:112-121 (2007). doi:10.1172/JCI28281. domains called sensitizers cannot activate BAX and BAK oligomerization directly $(8,14)$. Sensitizer BH3 domains instead contribute to cell death by competing for BCL2's hydrophobic BH3 domainbinding cleft, freeing activator $\mathrm{BH} 3$-only proteins to induce BAX and BAK oligomerization (Figure 1).

A cell-permeant compound, ABT-737, binds with high affinity to BCL2, BCL- $\mathrm{X}_{\mathrm{L}}$, and BCL-w, antagonizes their antiapoptotic function, and induces apoptosis in select human tumor cell lines, primary patient-derived cells, and a lymphoma mouse xenograph model $(14,15)$. While ABT-737 has been shown to be toxic to many cancer cells, including chronic lymphocytic leukemia (CLL) cells, the reason that some, but not all, cancer cells are sensitive to ABT-737 has remained obscure. In this study, we elucidate the reason that CLL cells are especially sensitive to antagonism of BCL2 function. We exploit a novel mitochondrial assay, which we call $\mathrm{BH} 3$ profiling, to examine the dependence of CLL cells on antiapoptotic proteins for survival. A mechanistic dissection shows that ABT-737 toxicity resides in its ability to function as a sensitizer, displacing the preexisting activator BIM from the BCL2, where it is constitutively sequestered. We furthermore show that $\mathrm{BH} 3$ profiling is an impressive predictor of sensitivity to ABT-737 in CLL cells and in myeloma cell lines. We identify the target molecular complexes disrupted by ABT-737 in CLL, predict cellular response to ABT-737, and provide a general model explaining BCL2 addiction in cancer cells. This understanding of the molecular basis of dependence on BCL2 and the action of ABT-737 in CLL and myeloma may provide useful tools for the clinical prediction of response to ABT-737 in a wide range of cancers.

\section{Results}

ABT-737 kills CLL cells in the low nanomolar range. ABT-737 is a cellpermeant small molecule that binds with high affinity to BCL2, 


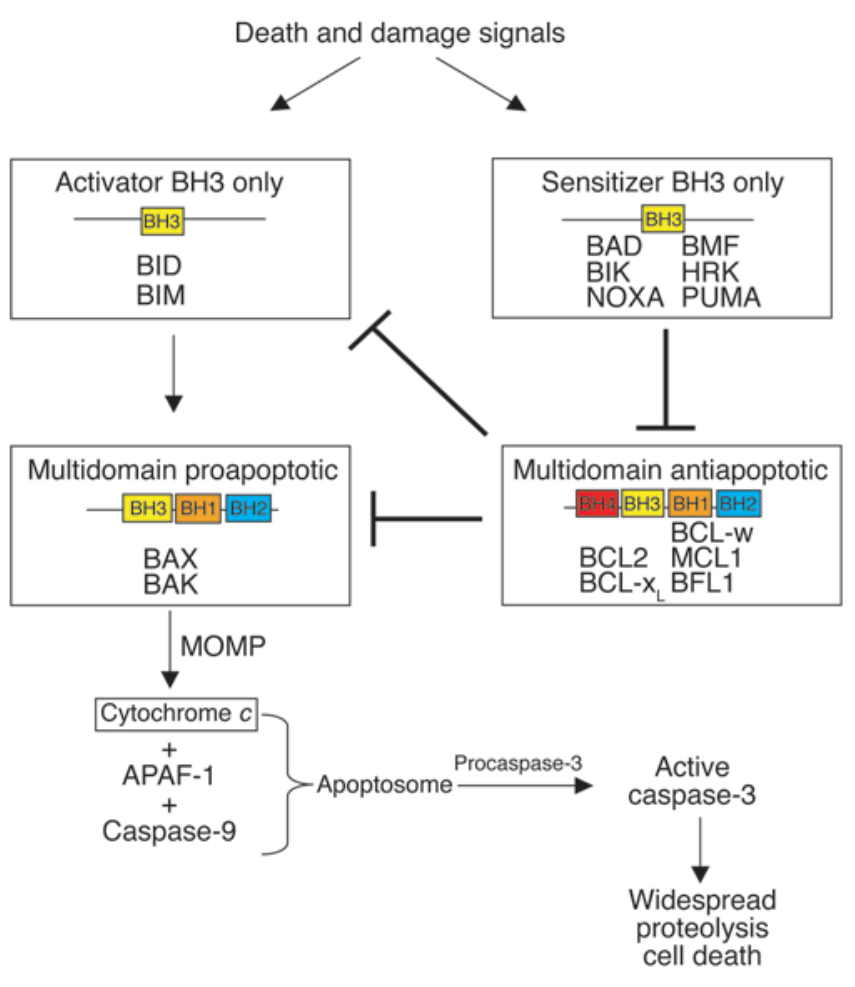

BCL- $\mathrm{X}_{\mathrm{L}}$, and BCL-w in the subnanomolar range. The negative control enantiomer is a stereoisomer of ABT-737 that binds to BCL2 with reduced affinity and has been used as a loss-offunction control $(14,15)$. CLL cells have shown sensitivity to ABT-737, so we initially selected them as a cancer model of BCL2 dependence (15). To test CLL cell sensitivity to ABT-737, freshly isolated primary CLL cells were incubated with ABT-737 or negative control enantiomer. After 48 hours, CLL cell death was assessed using annexin V staining. In all 24 CLL samples tested, apoptosis was induced within 48 hours by ABT-737 with an $\mathrm{EC}_{50}$ of $4.5 \pm 2.2 \mathrm{nM}$ (Figure 2A) (range 1.9-9.4 nM; see Supplemental

\section{Figure 1}

Schema of the intrinsic or mitochondrial programmed cell death pathway. In response to death signaling, activator $\mathrm{BH} 3-$ only proteins are triggered to interact with $\mathrm{BAX}$ and $\mathrm{BAK}$, inducing $\mathrm{BAX}$ and $\mathrm{BAK}$ oligomerization. This oligomerization is followed by MOMP, which releases proapoptotic factors such as cytochrome $c$ to the cytosol. Cytosolic cytochrome $c$ forms a complex with apoptosis proteaseactivating factor-1 (APAF-1) and caspase-9 to make the holoenzyme known as the apoptosome, which in turn activates effector caspase-3, leading to widespread proteolysis. This pathway can be interrupted by antiapoptotic members, such as BCL2, which can bind activator $\mathrm{BH} 3$-only proteins, preventing their interaction with $B A X$ and BAK. This inhibitory interaction can itself be antagonized by sensitizer $\mathrm{BH} 3-$ only domains, which compete for the binding site in BCL2, displacing activators bound by BCL2. BFL1, BCL2-related gene expressed in fetal liver.

Table 1; supplemental material available online with this article; doi:10.1172/JCI28281DS1). The negative control enantiomer was approximately 100 -fold less potent (mean $\mathrm{EC}_{50}$ : $574 \mathrm{nM}$ ) (Figure 2A). Directly targeting the apoptotic machinery might be expected to induce apoptosis rapidly. To test this prediction, we examined apoptosis in 5 CLL patient samples treated for 4 hours. These samples responded similarly to those receiving 48hour treatment (Figure 2B). Importantly, nonmalignant PBMCs from normal donors were resistant to $\mathrm{ABT}-737$, with an $\mathrm{EC}_{50}$ of more than $1000 \mathrm{nM}$ (Figure 2C).

Table 1 summarizes the clinical characteristics of the source patients in Figure 2A. We compared $\mathrm{EC}_{50}$ values between groups dichotomized by factors previously identified as prognostically useful in CLL. This analysis revealed that in no case did a difference in mean $\mathrm{EC}_{50}$ between groups exceed $2 \mathrm{nM}$. A nonparametric statistical comparison of the groups showed that none differed with statistical significance except the groups dichotomized by leukocyte count (Table 2). Thus, biological response to ABT-737 appears to be largely independent of traditional prognostic factors. These studies confirm that primary CLL cells are indeed sensitive to ABT-737, validating the use of primary CLL cells as a human cancer model for BCL2 dependence.
A

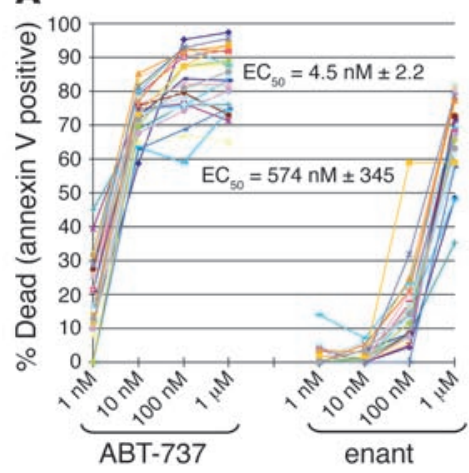

B

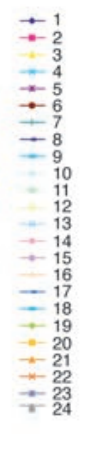

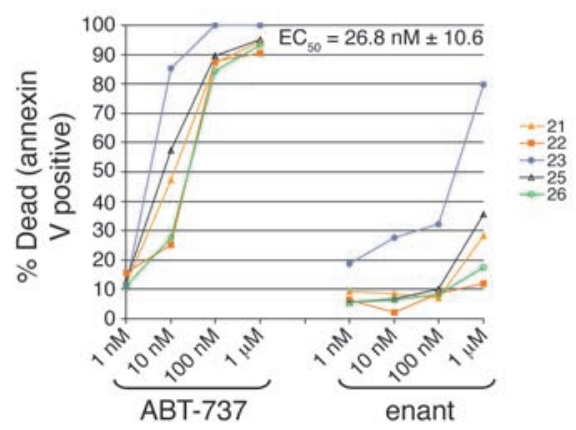

C



Figure 2

BCL2 antagonist ABT-737 efficiently kills primary CLL cells. (A) CLL cells from 24 patient samples (numbered at right) were cultured for 48 hours with different concentrations of ABT-737 or negative control enantiomer (enant). Death was quantitated by annexin $\mathrm{V}$ staining and normalized to controls treated with solvent (DMSO). The effective concentration at which $50 \%$ killing is observed ( $E C_{50}$ ) is shown. (B) CLL death induced by ABT-737 is rapid. CLL cells harvested from 5 patient samples (numbered at right) were cultured for 4 hours with different concentrations of compounds. Death was quantitated as in A. (C) Normal PBMCs are not sensitive to ABT-737. Four normal PBMC samples (numbered at right) were cultured for 24 hours in the presence of the indicated concentrations of compounds. Death was quantitated as in $\mathbf{A}$. 


\section{Table 1}

Characteristics of CLL patients contributing samples used in Figure 2A

\begin{tabular}{|c|c|}
\hline Characteristic & Value \\
\hline \multicolumn{2}{|l|}{ Rai stage $(n)$} \\
\hline 0 & 0 \\
\hline $1-2$ & 19 \\
\hline $3-4$ & 5 \\
\hline \multicolumn{2}{|l|}{$\operatorname{CD38}(n)$} \\
\hline Positive & 3 \\
\hline Negative & 4 \\
\hline Not assessed & 17 \\
\hline \multicolumn{2}{|l|}{ Treatment history $(n)$} \\
\hline Prior treatment & 3 \\
\hline Untreated & 21 \\
\hline \multicolumn{2}{|l|}{ IgVH status $(n)$} \\
\hline Mutated & 16 \\
\hline Not mutated & 5 \\
\hline Not assessed & 3 \\
\hline \multicolumn{2}{|l|}{ ZAP70 (n) } \\
\hline Positive & 6 \\
\hline Negative & 9 \\
\hline Not assessed & 9 \\
\hline Age, yr [median (range)] & $62.5(41-76)$ \\
\hline Leukocyte count, $\times 1000 / \mu \mathrm{l}[\text { median (range)] }]^{\mathrm{A}}$ & $133.3(47-502)$ \\
\hline LDH, U/I [median (range)] ${ }^{\mathrm{B}}$ & $487(185-2138)$ \\
\hline$\beta 2$ microglobulin (mg/ml) [median (range)] ${ }^{\mathrm{C}}$ & $3.1(1.7-5.5)$ \\
\hline
\end{tabular}

ANormal range, 4.8-10.8 × 1000/ul; Bnormal range, 313-618 u/l; Cnormal range, $0-2.7 \mathrm{mg} / \mathrm{ml}$.

BCL2 and BIM levels are consistent among CLL samples. Given the consistency of response to ABT-737, we wondered whether BCL2 protein expression was also uniform in CLL cells. Because the antiapoptotic BCL2 and the proapoptotic $\operatorname{BIM}(16,17)$ proteins are important determinants of commitment to apoptosis in lymphocytic cells, we tested the levels of these proteins in CLL cells. Western blots using antibodies specific for BIM and BCL2 revealed that BCL2 and BIM levels among 15 CLL cell samples were remarkably uniform (Figure 3A). Conversely, levels of BCL2 and BIM in PBMCs were consistently lower (Figure 3B).

To ensure that short-term culture did not affect BCL2 or BIM levels and perhaps alter response to ABT-737, protein lysates were made from CLL cell samples at the time of isolation and after 48 hours in culture. Neither BCL2 nor BIM levels changed during culture (Figure 3C). Because follicular lymphoma (FL) also overexpresses BCL2, albeit via the $t(14 ; 18)$ translocation, and exhibits sensitivity to ABT-737 (15), we compared BCL2 levels in CLL cells to levels in FL cells (Figure 3D). BCL2 levels were notably similar in the 2 diseases.

MCL1 and BID levels are low in CLL cells and likely have limited influence on the response to ABT-737. Next, we decided to determine the levels of MCL1 in CLL cells for several reasons: (a) MCL1 is an antiapoptotic relative of BCL2 expressed in CLL cells; (b) MCL1 can bind to BIM but is not antagonized by ABT-737 and might be expected to compensate for the loss of BCL2 function and maintain survival following ABT-737 treatment; (c) cleavage of MCL1 by caspases can allow release of BIM that can activate $\operatorname{BAX}(18,19)$; and (d) higher levels of MCL1 expression have been suggested as portending inferior clinical outcome $(20,21)$. To investigate the relative importance of MCL1 in maintaining survival in CLL cells, we quantitated amounts of MCL1 and BCL2 by Western blot (Figure 3E and Table 3). Using defined amounts of recombinant MCL1 and BCL2 proteins as quantitative protein standards, we found that MCL1 was expressed at levels 4- to 14-fold lower than those of BCL2 in CLL cells. To measure cleaved forms of MCL1, we prepared immunoblots of CLL whole-cell lysates either before or after treatment with ABT-737 and probed using an antibody that can detect cleaved forms of MCL1 (22). We found that cleaved forms of MCL1 were undetectable (Figure 3F and Supplemental Figure 1A). These results suggest that MCL1 in intact or in cleaved form is present at relatively low amounts compared with BCL2 in CLL cells and does not play an important role in determining cellular response to ABT-737.

Like BIM, BID has been identified as an activator BH3-only protein $(8,13,23)$. Therefore, we examined BID levels in CLL cells. Using an antibody that can detect either full-length or caspase8-cleaved p15 BID, we found that full-length p22 BID was barely detectable in whole-cell lysates. In addition, caspase-8-cleaved p15 BID was not detectable (Figure 3G). Treatment with ABT-737 did not induce increased levels of either p15 or p22 BID (Supplemental Figure 1B). Furthermore, neither form of BID was detectable as an immunoprecipitated complex with BCL2 (Supplemental Figure 1C). These data argue against an important role for BID in determining death following antagonism of BCL2 by ABT-737 in CLL cells.

$C L L$ mitochondria reveal a tonic dependence on BCL2 function to maintain outer membrane integrity. Since BCL2 opposes the mitochondrial pathway of apoptosis, we hypothesized that the toxicity of ABT-737 was based on a mitochondrial requirement for BCL2 function in CLL. We have characterized a panel of peptides derived from the $\mathrm{BH} 3$ domains of $\mathrm{BH} 3$-only proteins that behave as selective antagonists of BCL2 function in several genetically defined model systems $(8,14)$. For instance, $\mathrm{BH} 3$ peptides from $\mathrm{BAD}$, PUMA, BMF, and, with lower potency, BIK bind and inhibit BCL2 function whereas $\mathrm{BH} 3$ domains from NOXA, HRK, and BNIP-3A do not interact with BCL2. We have validated this panel with other antiapoptotic family members. The pattern of interaction is distinct for each antiapoptotic protein so that the function of each may be specifically detected by $\mathrm{BH} 3$ profiling.

\section{Table 2}

Comparison of clinical characteristics with in vitro sensitivity to ABT-737

\begin{tabular}{lcc}
\hline Parameter & \multicolumn{1}{c}{ Dichotomy } & $\boldsymbol{P}$ value \\
CD38 & Positive vs. negative & 0.11 \\
$\beta 2$ & $0-2.7$ versus $>2.7$ & 0.56 \\
Age & $0-55$ versus $>55$ & 0.14 \\
LDH & $0-618$ vs. $>618$ & 0.89 \\
wbc count & $0-133$ vs. $>133$ & $0.02^{\mathrm{A}}$ \\
Rai stage & $0-2$ vs. $3-4$ & 0.14 \\
Prior treatment & Yes vs. no & 0.93 \\
IgVH status & Mutated vs. not mutated & 0.10 \\
ZAP70 & Positive vs. negative & 0.69 \\
\end{tabular}

Statistical summary of dichotomized groups. Samples shown in Figure 2A were divided into groups based on their membership in 1 of the 2 classes for each property. Mann-Whitney $U$ test was used to determine whether the 2 groups differed in terms of statistical significance; this is summarized by the $P$ values in the third column. AHigher wbc count correlates with higher $\mathrm{EC}_{50}$. 
A

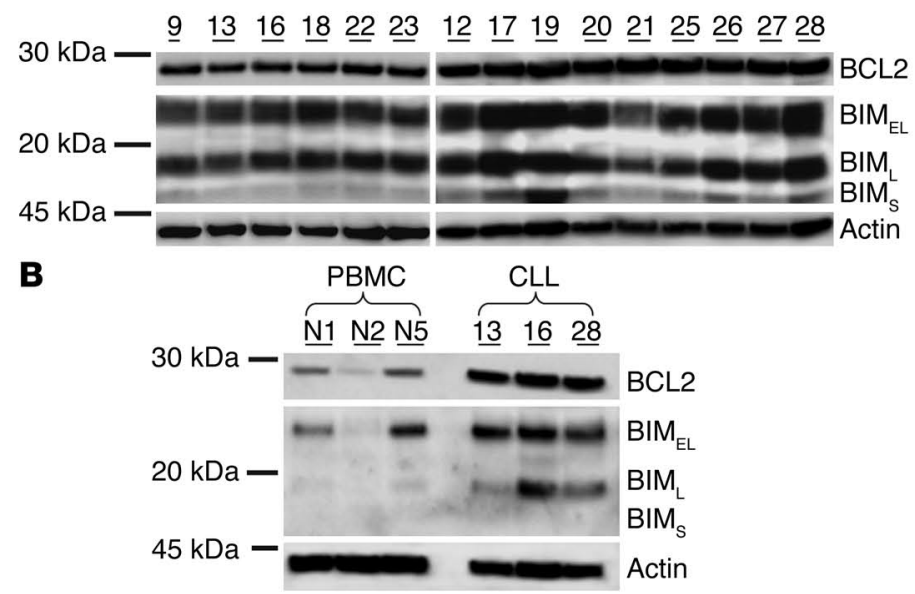

$\mathbf{E}$

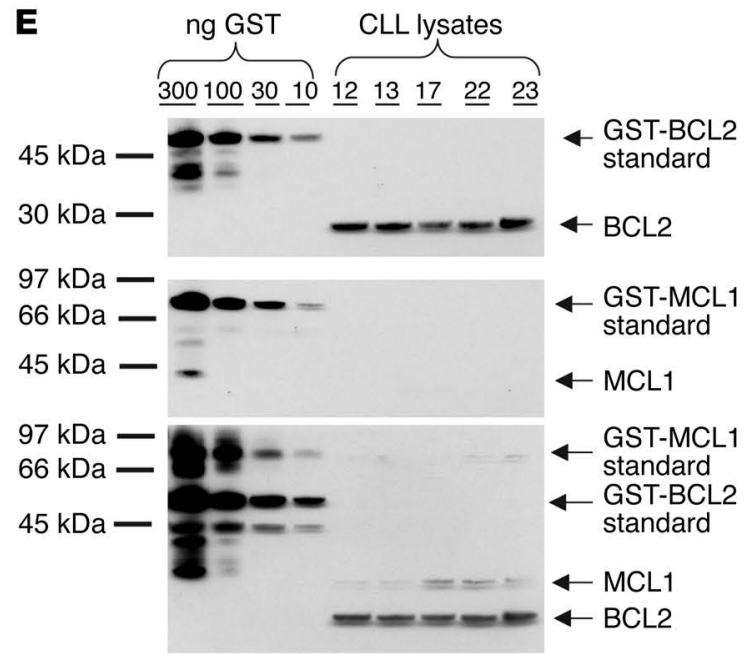

$\mathbf{F}$

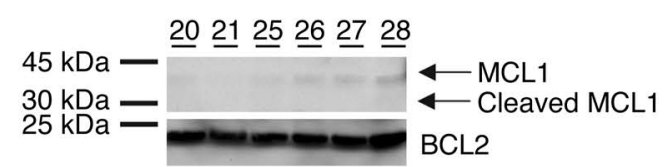

G



Figure 3

Protein expression in CLL cells. (A) BCL2 and BIM levels were uniform across 15 CLL samples tested by immunoblot of $5 \mu \mathrm{g}$ whole-cell lysates. Three isoforms of $B I M\left(B I M_{E L}, B I M_{L}\right.$, and BIMS) are shown. (B) BCL2 and BIM levels in PBMCs were lower than in CLL. Whole-cell lysates (5 $\mu$ g) evaluated by immunoblot. Three normal PBMC lysates are depicted at left, CLL lysates at right. (C) Short-term culture does not affect BCL2 or BIM protein levels. Two independent CLL lysates generated before and 48 hours after culture were probed for BCL2 and BIM. (D) BCL2 protein levels in 6 primary CLL cells (a-f) and primary FL cells are similar as seen by indexing immunoblots to lysates from the $t(14 ; 18)$-containing $H 2$ human lymphoma cell line. (E) Purified glutathione-S-transferase-tagged BCL2 (GST-BCL2) and GST-MCL1 (10-300 ng) were run next to 5 independent CLL lysates $(5 \mu \mathrm{g})$ to quantitate BCL2 and MCL1. MCL1 is detectable only upon long exposure (bottom panel). Relative amounts of BCL2 and MCL1 were calculated using densitometry and are depicted in Table 3. (F) Antibody detecting full-length and cleaved MCL1 does not demonstrate MCL1 cleavage products in CLL lysates. (G) CLL lysates $(10 \mu \mathrm{g})$ reveal low levels of BID and no detectable cleaved BID. DHL4 (DHL) cell lysate $(10 \mu \mathrm{g})$, known to express BID, and recombinant caspase-8-cleaved his-tagged BID (tB) (35 ng) were run as positive controls for antibody recognition. Note that the his-tag causes slower migration of uncleaved BID. (Numbers at top of gels correspond to patient sample numbers.)

Mitochondria that depend on BCL2 function for maintenance of their outer membrane integrity show induction of outer membrane permeability when treated with BAD, PUMA, and BMF but not NOXA, HRK, and BNIP-3A peptides. Therefore, CLL mitochondria were incubated with $\mathrm{BH} 3$ peptides as well as ABT-737 and negative control enantiomer (Figure 4). BAD, PUMA, and BMF induced cytochrome $c$ release whereas the NOXA, HRK, and BNIP-3A peptides and a point-null mutant of $\mathrm{BAD} \mathrm{BH} 3$ did not. This pattern is diagnostic of mitochondrial BCL2 dependence (14). ABT-737 also induced cytochrome $c$ release, validating that its target is located at the mitochondria of CLL cells and is required to prevent MOMP. Therefore, these $\mathrm{BH} 3$ profiling experiments demonstrate that CLL mitochondria depend on BCL2 function to maintain mitochondrial outer membrane integrity. Furthermore, these data elucidate a mechanism for the exquisite sensitivity of CLL cells to ABT-737 treatment. Finally, since the $\mathrm{BH} 3$ peptides in the sensitizer panel lack the ability to directly activate BAX and BAK, these experiments also implicated the presence of an activator molecule constitutively sequestered by BCL2 in CLL cells. CLL cells are therefore "primed for death" (14).
BIM bound to BCL2 primes CLL cells for killing by ABT-737. ABT-737 and the sensitizer $\mathrm{BH} 3$ peptides act as antagonists of antiapoptotic BCL2 but lack the ability to directly activate BAX and BAK. In order to induce MOMP, sensitizers require the presence of an activator, such as $\operatorname{BIM}$ or $\operatorname{BID}(8,14,15)$. The results above therefore suggest that an activator is bound to BCL2, then displaced by ABT-737 or the BCL2-binding BH3 peptides. Following displacement, we hypothesized, the freed activator could induce MOMP via interaction with BAX and BAK.

Since BIM and BCL2 were uniformly present in all CLL cell samples examined (Figure 3), we tested to determine whether BIM was indeed bound by BCL2. Using immunoprecipitation with a BCL2 antibody, we showed that BIM was consistently sequestered by BCL2 in primary CLL cells (Figure 5A). Furthermore, nearly all the cellular BIM pool in CLL cells existed in complex with BCL2 (Figure 5B). Reciprocal coimmunoprecipitation of BCL2 with BIM confirmed that much of BCL2 likewise existed in complex with BIM (Figure 5C). Similarly, in other cell types, including nonmalignant lymphocytes, other investigators have found nearly all cellular BIM sequestered by antiapoptotic proteins or at the mitochondrion $(24,25)$. To further investigate the sequestra- 


\section{Table 3}

Quantitation of BCL2 and MCL1 levels in primary CLL samples in Figure 3E

\begin{tabular}{lccc}
$\begin{array}{l}\text { CLL } \\
\text { lysate }\end{array}$ & $\begin{array}{c}\text { Molecules } \\
\text { BCL2/cell }\end{array}$ & $\begin{array}{c}\text { Molecules } \\
\text { MCL1/cell }\end{array}$ & $\begin{array}{c}\text { BCL2/MCL1 } \\
\text { ratio }\end{array}$ \\
12 & $8.1 \times 10^{6}$ & $6.4 \times 10^{5}$ & 13 \\
13 & $7.3 \times 10^{6}$ & $5.8 \times 10^{5}$ & 12 \\
17 & $3.6 \times 10^{6}$ & $9.9 \times 10^{5}$ & 4 \\
22 & $5.0 \times 10^{6}$ & $8.9 \times 10^{5}$ & 6 \\
23 & $9.2 \times 10^{6}$ & $6.5 \times 10^{5}$ & 14 \\
\hline
\end{tabular}

tion of BIM by mitochondrial BCL2, we examined the subcellular localization of BIM and BCL2 in the absence of detergents. Most $\mathrm{BIM}$ was present in the heavy membrane fraction that contained the mitochondria, with a smaller portion in the light membrane fraction (Figure 5D). Consistent with our immunoprecipitation results (Figure 5, B and C), BCL2 was distributed in a pattern similar to that of BIM. This finding offers further support for the tonic sequestration of BIM by BCL2 in CLL cells.

To determine whether ABT-737 could indeed displace BIM from BCL2, we treated BCL2 immunoprecipitates with ABT-737 and tested for the presence of BIM protein in the supernatant. Since ABT-737 is a $\mathrm{BH} 3$ domain mimetic and since we have found that interactions of short $\mathrm{BH} 3$ peptides with BCL2 are perturbed by the presence of detergent (data not shown), we performed these experiments in detergent-free conditions. ABT-737, but not the negative control enantiomer, displaced BIM from BCL2 into the supernatant (Figure 5E). $\mathrm{BIM}$ has been shown to activate BAX and BAK and induce their oligomerization $(8,26,27)$. If BIM displaced by ABT- 737 induces death by interacting with $\mathrm{BAX}$ and $\mathrm{BAK}$, then oligomerization of $\mathrm{BAX}$ and BAK should be observed following ABT-737 treatment. Examination of protein lysates from mitochondrial preparations revealed the presence of mitochondrial BAX and BAK (Figure 5F). Consistent with our model, both BAX and BAK formed oligomers within 4 hours of treatment of CLL cells with ABT-737 (Figure 5G).

Next, we attempted to independently validate the model that ABT-737 releases BIM from BCL2 and promotes BAX and BAK multimers, which lead to induction of MOMP and cytochrome $c$ release. If BIM is indeed required for inducing MOMP following BCL2 antagonism, selective sequestration of BIM should cause a reduction in cytochrome $c$ release following antagonism of BCL2 on CLL mitochondria. As shown in Figure 5H, we antagonized BCL2 function with the sensitizer BAD BH3 peptide. As shown previously in Figure 4, BAD BH3 by itself induced cytochrome $c$ release. However, addition of an antibody that binds the $\mathrm{BH} 3$ domain of BIM caused a dramatic reduction in cytochrome $c$ release induced by BAD BH3. An irrelevant antibody had no effect. Prevention of cytochrome $c$ release by masking BIM supports a model in which antagonism of ABT-737 is toxic to CLL cells due to the displacement of BIM (Figure 5E) from a BCL2/BIM complex. Displaced BIM then induced BAX and BAK oligomerization (Figure 5G), MOMP, and commitment to programmed cell death. An important implication of these results is that BCL2 expression is necessary but not sufficient to dictate response to antagonism of BCL2 by ABT-737 or sensitizer BH3 peptides. Activator BH3only proteins, such as BIM, must be sequestered by BCL2 for the cell to be sensitive to BCL2 antagonism.
$B H 3$ profiling discriminates $B C L 2$ versus $M C L 1$ requirement in myeloma cells. $\mathrm{BH} 3$ profiling revealed a requirement for BCL2 in CLL, which is reflected at the cellular level by sensitivity to ABT-737. To determine whether BH3 profiling could predict antiapoptotic requirements in other human malignancies, we examined human multiple myeloma cell lines LP1 and L363. When mitochondria isolated from LP1 were profiled, cytochrome $c$ release was induced by $\mathrm{BH} 3$ domains of BIK, NOXA, PUMA, and BMF but not BAD, HRK, and BNIP-3A (Figure 6A). This interaction pattern is inconsistent with BCL2 dependence; rather, it matches that of $\operatorname{MCL} 1(9,14,28)$. Mitochondria from L363 cells, in contrast, revealed a pattern of sensitivity to BH3 domains of BAD, PUMA, BMF, and BIK but not NOXA, HRK, or BNIP-3A (Figure 6A). As described above and shown in Figure 2, this profile suggests a BCL2 requirement. It is notable in this context that LP1 cells showed significantly greater sensitivity to antisense oligonucleotide downregulation of MCL1 than did L363 cells (29). We examined expression levels of MCL1 and BCL 2 in these 2 cell lines, and found, consistent with our mitochondrial functional data, more BCL2 present in L363 than in LP1, but more MCL1 in LP1 than L363 (Figure 6B). In both cases, ample BIM was present to provide the activator function needed to signal BAX and BAK to induce MOMP.

If mitochondrial $\mathrm{BH} 3$ profiling predicts cellular behavior and sensitivity to ABT-737, then L363 cells, but not LP1 cells, should be sensitive to antagonism of BCL2 by ABT-737. To test this prediction, we treated cells with ABT-737 and negative control enantiomer and found that L363 cells were vastly more sensitive to ABT-737 than were LP1 cells (Figure 6C).

Both L363 and CLL cells demonstrated sensitivity to ABT-737 and expression of BIM. The sensitivity of both cell types to this compound and to peptides that antagonize BCL2 in BH3 profiling suggests the presence of at least 1 activator $\mathrm{BH} 3$-only protein sequestered by BCL2. To formally determine whether BIM

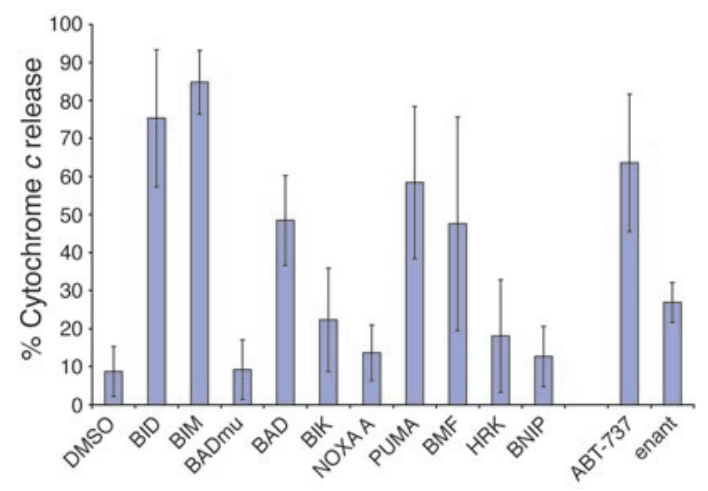

\section{Figure 4}

$\mathrm{BH} 3$ profiling of CLL mitochondria reveals BCL2 dependence. Mitochondria were isolated from independent primary CLL patient samples and treated with BH3-only domain peptides as indicated $(100 \mu \mathrm{M})$ or ABT-737 or negative control enantiomer $(100 \mathrm{nM})$. DMSO $(1 \%)$ is a solvent control. Cytochrome $c$ release was analyzed via ELISA. Note that activators $\mathrm{BID}$ and BIM BH3 peptides interact with all antiapoptotic proteins tested and furthermore can directly activate BAX and BAK (8) so that they act as positive controls for cytochrome $c$ release assays. BADmu, a point mutant of the BAD BH3-only domain, was used as a negative control. $n=7$, except for BADmu, where $n=5$, and ABT-737 and negative control enantiomer, where $n=3$. Error bars represent SD. 
A



D
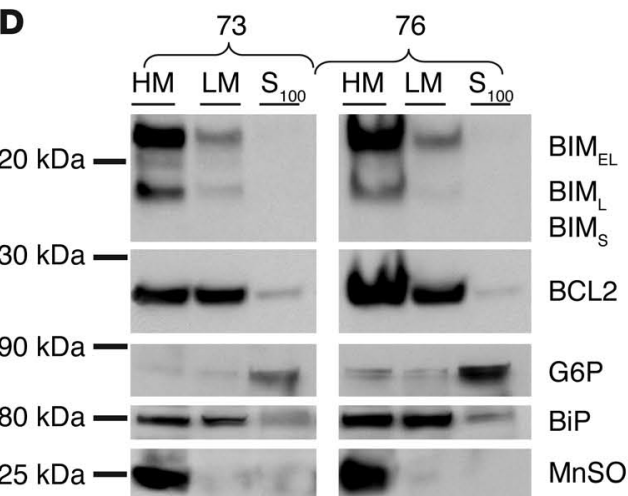

B

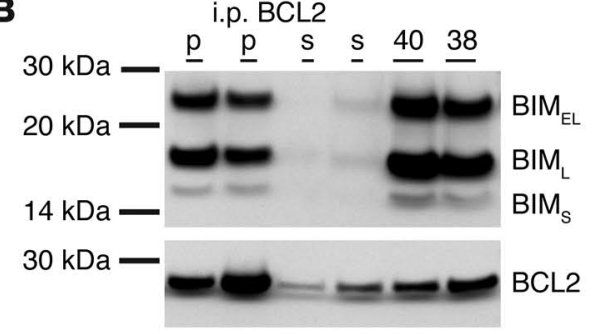

76

$\overbrace{\text { HM LM S } S_{100}}^{76}$

E

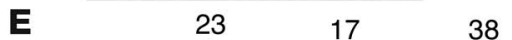

$\mathrm{BIM}_{\mathrm{EL}}$ BIM $_{L}$ $\mathrm{BIM}_{\mathrm{S}}$

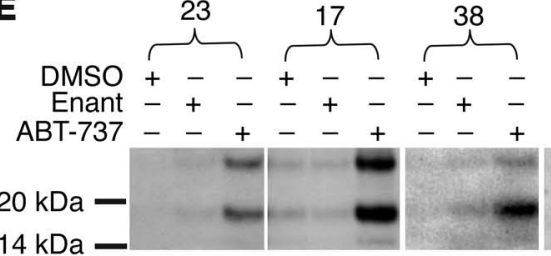

C



$\mathbf{F}$


$23 \mathrm{kDa}$ - BAX

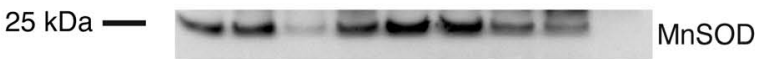

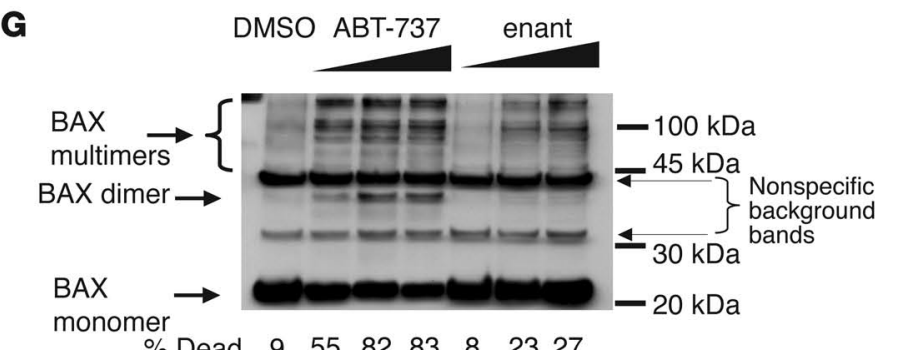

MnSOD

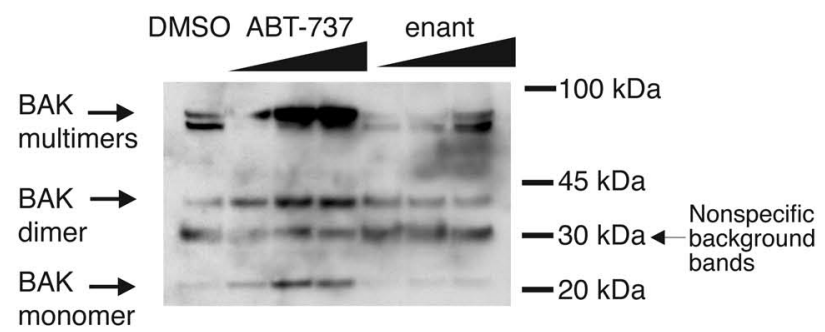

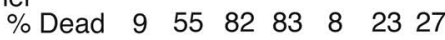

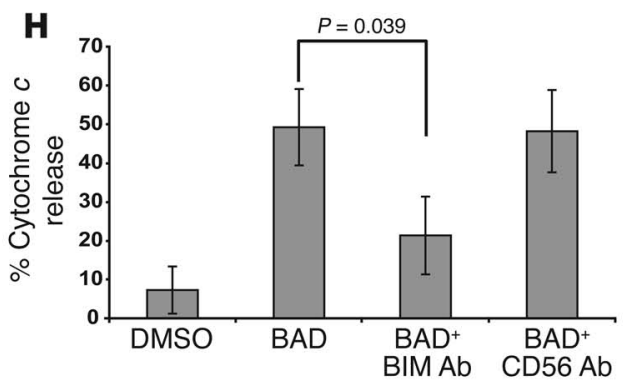

Figure 5

$\mathrm{BIM}$ is sequestered by BCL2 and displaced by antagonism of BCL2. (A) Anti-BCL2 immunoprecipitation of CLL whole-cell lysates (50 $\mu \mathrm{g})$. (B and C) CLL whole-cell lysates $(50 \mu \mathrm{g})$ immunoprecipitated with anti-BCL2 (B) or anti-BIM (C) antibodies. Immunoprecipitation pellet (p), supernatant (s), and $100 \%$ of total lysate used for immunoprecipitation were loaded (40, 38 [B]; 38 [C]). (D) Subcellular fractions of primary CLL cells (50 $\mu$ g) were immunoblotted for BIM and BCL2. Fractionation verified with marker proteins glucose 6 phosphate dehydrogenase (G6P), ER-binding protein (BiP), and mitochondrial superoxide dismutase (MnSOD). HM, heavy membrane; LM, light membrane. (E) Anti-BCL2 immunoprecipitation from $50 \mu \mathrm{g}$ CLL lysates (Triton X-100 used for 2 samples at left, CHAPS for 2 at right). Incubation with DMSO (1\%), $1 \mu \mathrm{M}$ negative control enantiomer, or $1 \mu \mathrm{M}$ ABT-737 displaced BIM to the supernatant identified by anti-BIM immunoblot. (F) Lysates of CLL mitochondria (15 $\mu \mathrm{g}$ ) in RIPA buffer were immunoblotted for BAK, BAX, and MnSOD (loading control). DHL4 lysate (15 $\mu \mathrm{g}$ ) was used as a positive control for BAK. Patient sample numbers shown at top of blots in $\mathbf{A}-\mathbf{F}$. (G) CLL cells were incubated with DMSO $(0.1 \%)$, ABT-737, or negative control enantiomer $(10 \mathrm{nM}, 100 \mathrm{nM}$, or $1 \mu \mathrm{M})$ for 4 hours. Percentage of cells that were dead was assessed by annexin V. Oligomerization of BAX and BAK evaluated by anti-BAX or anti-BAK immunoblot of chemically cross-linked whole-cell lysates. (H) Mitochondria isolated from CLL samples were incubated with DMSO $(1 \%)$ or BAD BH3 peptide $(100 \mu \mathrm{M})$. Samples were preincubated with antibodies directed against either the human BIM BH3 domain or irrelevant antigen (CD56). $n=5$. Error bars show \pm SD.

primes these cells for death and provokes sensitivity to ABT737, we silenced BIM expression using a lentiviral short hairpin RNA (shRNA) construct. The BIM lentiviral shRNA construct reduced BIM mRNA levels (data not shown) as well as protein levels of all 3 BIM isoforms (Figure 6D). Cells with reduced BIM levels showed a significant reduction in sensitivity to ABT-737 

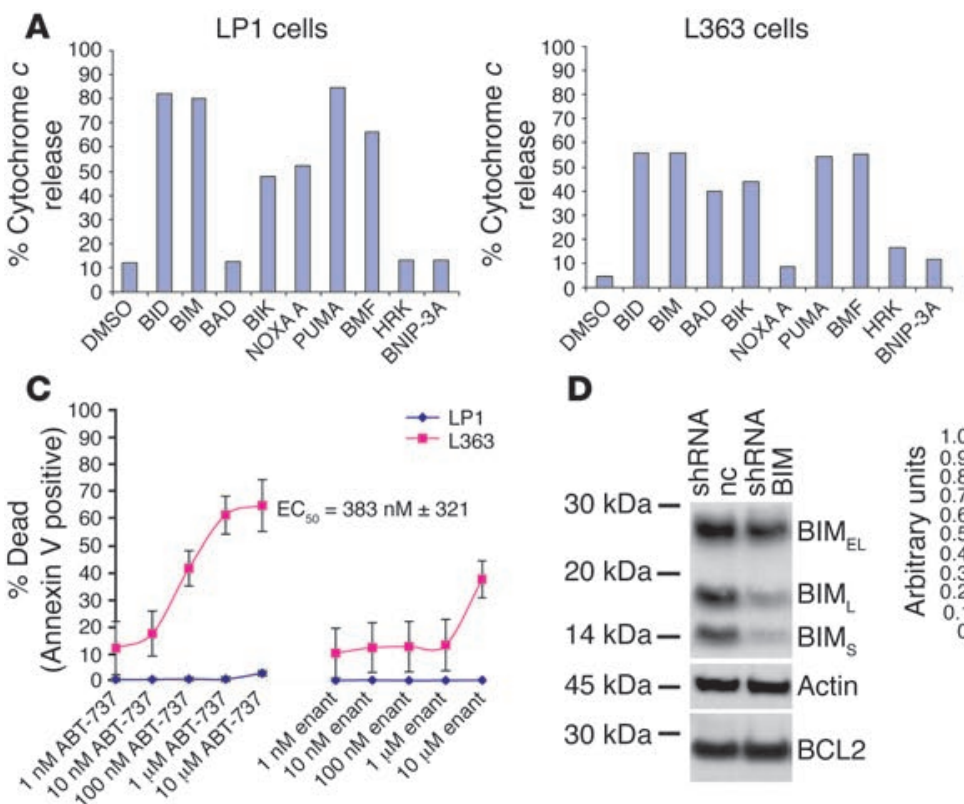

D

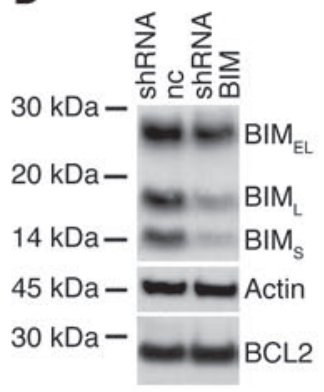

B



$\mathbf{E}$

\section{Figure 6}

BH3 profiling correctly predicts ABT-737 sensitivity in myeloma cell lines. (A) Mitochondria isolated from LP1 cells treated with $100 \mu \mathrm{M} B \mathrm{~B} 3 \mathrm{peptides}$ show an MCL1-dependent pattern of cytochrome $c$ release. Mitochondria isolated from L363 cells display a BCL2-dependent pattern. (B) Lysates $(10 \mu \mathrm{g})$ of LP1 and L363 cell lines subjected to Western blot, comparing levels of MCL1, BCL2, and BIM. (C) L363 cells are sensitive and LP1 cells resistant to 48-hour treatment with $\mathrm{ABT}-737$ and negative control enantiomer. $n=3$. Error bars show \pm SD. (D and E) Reduction of BIM confers resistance to ABT-737. BIM levels in L363 cells were reduced using lentiviral-delivered shRNA against exon 5 of BIM. (D) Western blot demonstrating knockdown of BIM in shRNA BIM-treated cells compared with negative control shRNA luciferase-treated (nc) cells. BCL2 levels are also shown at bottom. Quantification of protein bands by densitometry reveals $40 \%$ total BIM knockdown (white bars) with more than half of BIM $\mathrm{L}_{\mathrm{L}}$ and BIM $\mathrm{S}_{\mathrm{S}}$ isoforms reduced, as compared with shRNA luciferase (black bars). Samples were normalized to actin. (E) L363 cells infected with lentiviruses expressing shRNA BIM are less sensitive to 48-hour treatment with ABT-737 $(1 \mu \mathrm{M})$ than L363 cells infected with lentiviruses expressing shRNA luciferase. These data indicate that the presence of BIM complexed with BCL2 is responsible for ABT-737 sensitivity. $n=3$. Error bars show \pm SD.

(Figure 6E). These data further support the critical role of BIM in conducting death signals following displacement from BCL2 by ABT-737.

These data offer promising confirmation that cellular dependence on BCL2 or any other individual antiapoptotic protein can be predicted based on mitochondrial sensitivity to our $\mathrm{BH} 3$ peptide panel, BH3 profiling. They furthermore suggest that myeloma cases may be discriminated and perhaps differentially targeted based on dependence on either BCL2 or MCL1.

\section{Discussion}

It has been hypothesized that cancer cells, due to their numerous physiologic aberrancies, require a block in the normal apoptotic pathways in order to survive $(30,31)$. Overexpression of antiapoptotic BCL2 family members, such as BCL2 and MCL1, has been documented in cancer cells over the last few decades, but the key therapeutic question of whether these proteins are required for survival has remained largely unanswered.

Recent description of a micro-RNA-based mechanism by which CLL cells express high levels of BCL2 (32) combined with development of a small molecule antagonist of BCL2 (15) make timely a mechanistic investigation into the role of BCL2 in CLL cell maintenance. Attempts to target BCL2 in CLL cells using an antisense oligonucleotide strategy have yielded relatively modest reductions in BCL2 protein and modest clinical benefit (33). Thus, these studies have not been well suited to studying the requirement for BCL2 in CLL.
We have described a strategy called $\mathrm{BH} 3$ profiling and validated its ability to diagnose addiction to antiapoptotic BCL2 proteins in many model systems (14). Prior to these studies, it was unclear whether $\mathrm{BH} 3$ profiling could determine dependence on antiapoptotic BCL2 family proteins in primary human cancer cells. Here, we introduce the use of $\mathrm{BH} 3$ profiling to determine antiapoptotic protein dependence in primary cells of a human cancer, CLL. We found that $\mathrm{BH} 3$ profiling detects a dependence on BCL2 in CLL that accurately predicts sensitivity to the small molecule inhibitor of BCL2, ABT-737. This test of BH3 profiling on primary human cancer cells shows that $\mathrm{BH} 3$ profiling is a unique and powerful technique for the prediction of sensitivity to agents that antagonize function of antiapoptotic BCL2 family members. BH3 profiling furthermore correctly detected the existence of CLL in a primed state, a validation of the ability of $\mathrm{BH} 3$ profiling to probe apoptotic pathways of primary cancer cells. The robustness of our results supports the application of $\mathrm{BH} 3$ profiling to the study of apoptotic pathways to any cell of interest, malignant or nonmalignant, primary cell or cell line.

As predicted by the $\mathrm{BH} 3$ profiling results, which showed a consistent pattern of BCL2 dependence, the BCL2 antagonist ABT-737 was toxic to all primary CLL cell samples tested in these studies. The uniformity of this toxicity was impressive given the range of clinical properties, including stage, history of prior treatment, and prognostic indicators, of the samples tested. The uniformity of in vitro response was mirrored in the striking uniformity of BCL2 


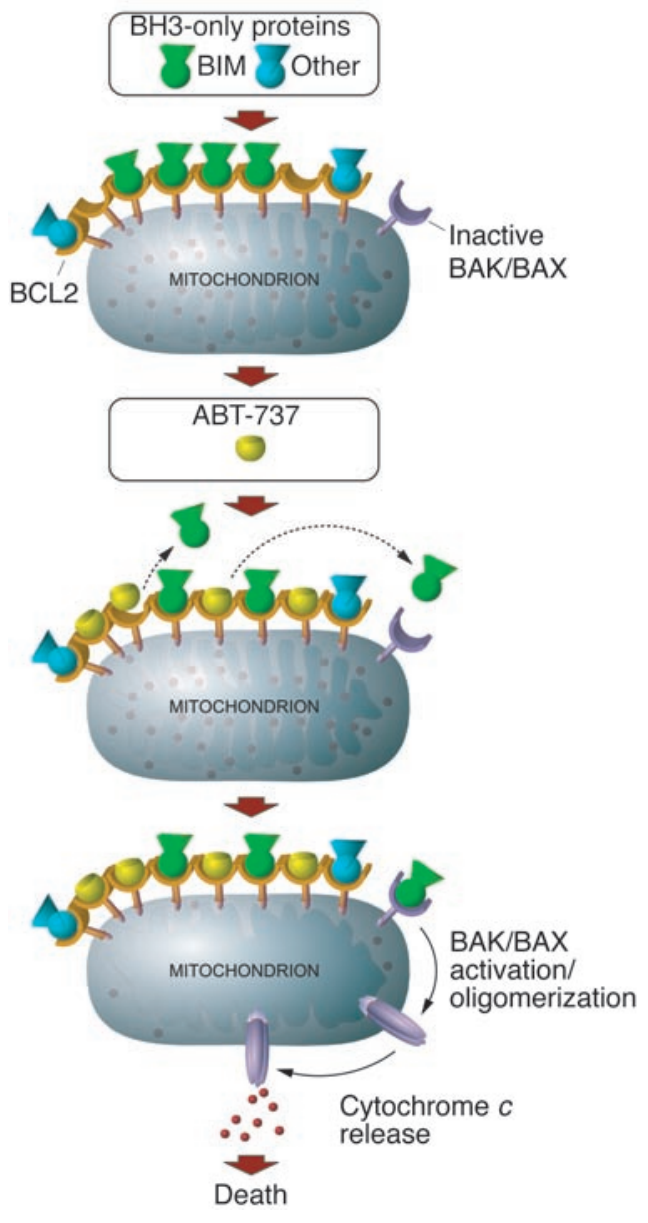

Figure 7

Model of ABT-737-induced death at the mitochondria. Mitochondrial BCL2 sequesters BIM in CLL cells. Upon addition of ABT-737, BIM is displaced and BCL2 becomes occupied by ABT-737. Freed BIM then interacts with $\mathrm{BAX}$ or $\mathrm{BAK}$, inducing oligomerization leading to cytochrome $c$ release and irreversible commitment to programmed cell death. $\mathrm{BCL} 2$ primed with activator $\mathrm{BH} 3-$ only proteins renders the cancer cell sensitive to treatment with ABT-737 and possibly other chemotherapeutic agents.

and BIM expression in CLL cells. It is interesting to note that the level of BCL2 in CLL cells is quite similar to that in FL cells, provoking conjecture that a fairly narrow range of BCL2 expression is optimal for oncogenesis and tumor maintenance.

MCL1 levels have been studied as a prognostic indicator in CLL, and it has been suggested that high MCL1 expression correlates with a decreased chance of complete response to chemotherapy $(20,21)$. As in previous reports, we found that MCL1 is difficult to detect by immunoblot. By using loading standards of recombinant proteins, we demonstrated that MCL1 levels are much lower than BCL2 levels. This offers an explanation of why MCL1 expression is apparently unable to compensate for the loss of BCL2 function after antagonism by ABT-737. There simply may not be sufficient free MCL1 available to bind the BIM that is displaced. It should be noted, however, that the vast majority of the samples studied came from patients who had not received treatment. It may be that other subsets of CLL express greater amounts of MCL1. Furthermore, our results do not rule out the possibility that higher MCL1 expression might be selected for by chemotherapy treatment or long-term BCL2 antagonism. Nonetheless, the extent to which dependence on BCL2 outweighs dependence on MCL1 in the CLL cell samples we studied is striking.

CLL cell death from ABT-737 occurred rapidly, as early as 4 hours after treatment. This suggests that BCL2 antagonism engages an apoptotic pathway that is already poised to respond in CLL cells. In molecular terms, this is consistent with our observation that BCL2 tonically sequestered and stored prodeath BIM, whose displacement is required for mitochondrial apoptosis following BCL2 antagonism. In support of this model, we show that ABT-737 displaced BIM from BCL2 (Figure 5E). Freed BIM was then required to signal downstream death events, including BAX and BAK oligomerization (Figure 5G), as demonstrated by the BIM-masking experiment (Figure $5 \mathrm{H}$ ) and our BIM-knockdown experiment (Figure 6, D and E). Our knockdown data indicate a $40 \%$ reduction in BIM protein levels (Figure 6D) and a $30 \%$ decreased sensitivity to ABT-737 (Figure 6E). These results suggest a BIM dosage dependence for downstream proapoptotic events. Preliminary results suggest that ABT-737 competition for binding to the BCL2 pocket may be perturbed by the presence of detergent. This conjecture can be investigated in the future by biophysical methods, such as ${ }^{15} \mathrm{~N}-\mathrm{HSQC} \mathrm{NMR}$, a nuclear magnetic resonance technique that can detect interaction of molecules in solution.

The paucity of BID and caspase-cleaved BID in total and in complex with BCL2 (Figure 3G and Supplemental Figure 1, B and C) suggests that the BH3-only activator BID does not play such an important role in mediating death from ABT-737. Although BIM plays a demonstrably critical role in ABT-737-induced death, we cannot exclude the participation of other uncharacterized (and perhaps undiscovered) BH3-only molecules. Nevertheless, an important consequence of these results is that the presence of BCL2 alone does not determine sensitivity to BCL2 antagonism. Sensitivity also requires the presence of an activator molecule, likely triggered by aberrancies of the malignant phenotype, including genomic instability, oncogene activation, and cell-cycle checkpoint violation (Figure 7).

In CLL cells, we found that almost all BIM appeared to be present in complex with BCL2. Early experiments suggested that BIM can be found complexed with dynein light chain and microtubules but could be displaced from this niche to BCL2 under apoptotic stimuli (34). It may well be that CLL cells are subject to tonic apoptotic stimuli due to their malignant phenotype, thus displacing BIM from a niche it might otherwise occupy. Alternatively, the "normal" niche for BIM may vary according to cell type, a view supported by more recent reports of BIM localization $(24,25)$.

One might then expect a natural therapeutic window to exist between cancer cells bearing primed BCL2 and normal cells with BCL2 that is relatively unoccupied by activators, due to the absence of tonic-death signaling. We and others have tested many nonmalignant cell types and found them resistant to antiapoptotic inhibition, suggesting that priming of antiapoptotic molecules with activator $\mathrm{BH} 3$-only proteins may be found selectively in cancer cells $(8,14,15,35)$. In fact, BCL2 antagonism by ABT-737 is relatively nontoxic to normal PBMCs (Figure 2C) and to the vast majority of tissues in mice (15). When mice in a tumor xenograft experiment were treated with doses as high as $100 \mathrm{mg} / \mathrm{kg} / \mathrm{d}$ for 21 days, mice maintained their weight, and only thrombocytopenia and lymphopenia were noted. However, at doses less than $30 \mathrm{nM}$, most CLL cells die within 4 hours in vitro. 
The uniformity of the toxicity, irrespective of traditional negative prognostic factors, suggests that directly targeting the death machinery may be a way to short-circuit mechanisms for resistance to conventional chemotherapy that likely act further upstream of the commitment to apoptosis.

$\mathrm{BH} 3$ profiling provides the distinct benefit of making it possible to study the physiology of freshly isolated primary cancer cells unaltered by ex vivo culture. Traditionally, physiologic analysis of primary cancer cells has suffered from the requirement for ex vivo culture. Many cancer cells survive poorly in culture, and surviving cells may undergo changes in gene expression patterns or clonal evolution of new genetic changes that fundamentally alter the cell being studied. Probably due to these problems, correlation of in vitro-cultured tumor cell chemotherapy response with in vivo tumor chemotherapy response has been inadequate to warrant introduction of in vitro sensitivity assessment into clinical practice. Using $\mathrm{BH} 3$ profiling, we extracted critical information about the apoptotic pathway in CLL cells as it exists in vivo. BH3 profiling revealed a mitochondrial requirement for an antiapoptotic protein. The specific pattern of $\mathrm{BH} 3$ peptide-induced cytochrome $c$ release correctly implicated BCL2 as the critical protein for maintenance of mitochondrial outer membrane integrity. Furthermore, this correctly predicted the sensitivity of CLL cells to BCL2 antagonism by ABT-737. Finally, this strategy correctly implicated the presence of an additional activator protein, subsequently identified as BIM.

Confirming the utility of this strategy, $\mathrm{BH} 3$ profiling also correctly identified a dependence on MCL1 in LP1 and a dependence on BCL2 in L363 myeloma cells. These mitochondrial results were confirmed at the cellular level by sensitivity to MCL1 knockdown for LP1 (29) and by sensitivity to BCL2 antagonism by ABT-737 for L363 (Figure 6).

The function of BCL2 as an antiapoptotic molecule was initially determined using overexpression models, and subsequent study of its function has largely depended on overexpression models $(36,37)$. In cells that have already established equilibrium with their environment, forced expression of additional BCL2 provides extra antiapoptotic reserve that reproducibly buffers cells from a wide variety of chemical, radiological, and biological insults. However, our data indicate that expression of BCL2 may not provide additional antiapoptotic reserve in cancer cells. We suggest that when BCL2 overexpression is selected for during oncogenesis, it is selected for by the pressures of ongoing tonic-death signaling that is activated by numerous behaviors common to the cancer phenotype, including genomic instability, oncogene activation, and cell-cycle checkpoint violation. In surviving cancer cells, this selected-for BCL2 is therefore likely to be tonically filled with prodeath molecules, such as BIM. This creates an ongoing requirement for BCL2 function and provides little, if any, additional antiapoptotic reserve to buffer chemotherapeutic insults. In fact, by sequestering significant amounts of activators, a cancer cell found to express BCL2 may instead be primed for apoptosis, ready to commit to programmed cell death following minimal challenges to its limited antiapoptotic reserve. We therefore suggest that BH3-only priming of BCL2 offers an explanation for the notable (and seemingly paradoxical) chemosensitivity of cancers, such as CLL or FL, that are archetypes of abundant BCL2 expression in cancer. In addition, it may be that similar priming of antiapoptotic BCL2 family members offers an explanation in certain cases for the otherwise often obscure mechanisms behind preferential sensitivity of many cancer cells to chemotherapeutic or radiotherapeutic challenges. BH3-only priming of BCL2 certainly offers an explanation for sensitivity to an antagonist of BCL2, such as ABT-737.

In summary, we introduce a strategy, $\mathrm{BH} 3$ profiling, which acts as a powerful probe of the apoptotic pathway in primary cancer cells. BH3 profiling accurately predicts dependence on individual antiapoptotic proteins. It furthermore predicts sensitivity to ABT-737 and has the potential to predict sensitivity to other antagonists of antiapoptotic proteins as they arise. It correctly implicated the presence of an activator BH3-only protein in CLL, which we subsequently identified as BIM. As it requires no ex vivo cell culture, $\mathrm{BH} 3$ profiling can provide critical information about apoptotic regulation in essentially any cell, including primary cancer cells.

\section{Methods}

Isolation and short-term culture of human cells. Blood $(15 \mathrm{ml})$ in heparin-treated tubes was obtained from each anonymous CLL patient and processed without freezing. Equal volume of media (RPMI medium, 10\% human bovine serum, supplemented with $10 \mu \mathrm{g} / \mathrm{ml}$ insulin and $10 \mathrm{mg} / \mathrm{ml}$ transferrin) was mixed with each sample and CLL cells isolated by centrifugation through Ficoll-PAQUE Plus (Amersham Biosciences). Cells were washed twice in media and cultured at a density of $2.0 \times 10^{6}$ cells $/ \mathrm{ml}$ for up to 48 hours. Samples used for Figure 2A were obtained from 24 patients identified with a wbc count of more than $50,000 / \mu 1$. We conformed to all guidelines and regulations in accordance with Internal Review Board protocols 99-224 (Dana-Farber Cancer Institute). These experiments were approved by the Dana-Farber Cancer Institute Internal Review Board, and clinical material was obtained from subjects, who gave informed consent. Normal PBMCs were obtained from tubing discarded following platelet donation by anonymous normal donors and processed as above, except cultured without insulin and transferrin.

CLL clinical criteria. FISH analysis was performed by the Brigham and Women's Hospital cytogenetics laboratory using a CLL panel of multicolor probe sets (Vysis; Abbott) (38). CLL cells were processed and IgVH and ZAP70 status was determined by the CLL Research Consortium Tissue core, using previously established methods (39). Somatic hypermutation in the IgVH locus was classified as absent when $98 \%$ or greater homology to germline was measured. Patient samples were classified as ZAP70 positive when at least $20 \%$ of cells were positive and CD38 positive when at least $30 \%$ of cells were positive. In Table 2, $\beta 2$ microglobulin and LDH were dichotomized by normal or abnormal, while age and wbc count were dichotomized at the mean.

Annexin $V$ assay. Cells were stained with fluorescent conjugates of annexin $\mathrm{V}$ (BioVision) and propidium iodide and analyzed on a FACSCalibur machine (BD).

Statistics. Experimental replicates were performed using lysates or mitochondria from different CLL samples. $P$ values were obtained with a 2 -tailed Student's $t$ test, and $P<0.05$ was considered statistically significant. GraphPad Prism software version 4 was used to determine $\mathrm{EC}_{50}$ values by nonlinear dose-response curve fitting and to perform MannWhitney nonparametric testing shown in Table 2.

$B A X$ and BAK oligomerization. Freshly isolated CLL cells $\left(10^{7}\right)$ were incubated with DMSO, and $10 \mathrm{nM}, 100 \mathrm{nM}$, or $1 \mu \mathrm{M}$ ABT-737 or negative control enantiomer for 4 hours. The percentage of cells that died was assessed by annexin $\mathrm{V}$ staining and FACS analysis. Cells were treated with $0.3 \%$ saponin and $10 \mu \mathrm{M}$ bismaleimidohexane for 30 minutes on ice. Cells were then lysed, loaded onto a $10 \%$ BIS-Tris polyacrylamide gel, transferred, and immunoblotted for BAX or BAK. 
BIM knockdown by shRNA. The plasmids PCMVDR8.91, pMD.G, and pLKO.1puro-luciferase and PLKO.1puro-BIM were obtained from the RNAi Consortium. Retroviral supernatants were produced by cotransfection of 293T cells with pCMVAR8.91, pMD.G, and either pLKO.1puro-Bim or pLKO.1puro-luciferase (40). L363 cells $\left(5 \times 10^{6}\right)$ were incubated with retroviral supernatant for 4 hours. Infected cells were then grown in $0.5 \mu \mathrm{g} / \mathrm{ml}$ puromycin for 2 weeks. The following oligonucleotides, directed against exon 5 of human BIM, were used to create the pLKO.1puro-BIM plasmid: forward, 5'-CCGGATGGTTATCTTACGACTGTTACTCGAGTAACAGTCGTAAGATAACCATTTTT-3'; and reverse, 5' - AATTAAAAAATGGTTATCTTACGACTGTTACTCGAGTAACAGTCGTAAGATAACCAT-3'.

The names of peptides used in experiments and the methods for multiple myeloma cell culture, cytochrome $c$ release, immunoblots, immunoprecipitation, Western blot protein quantification, and subcellular fractionation can be found in Supplemental Methods.

\section{Acknowledgments}

The authors wish to acknowledge graphical assistance from Eric Smith; technical assistance from Gullu Gorgon and Caroline Chen; helpful discussions with Stanley Korsmeyer, John
Gribben, Bruce Chabner, and Glenn Dranoff; the kind gift of ABT-737 and negative control enantiomer compounds from Saul Rosenberg of Abbott Laboratories; and IgVH and ZAP70 results provided by the CLL Research Consortium Tissue core. This work has been supported by funding from NIH grant K08 CA10254, the Dunkin' Donuts Rising Stars Program, the Richard and Susan Smith Family Foundation, and the Sidney Kimmel Foundation for Cancer Research. C.D. Novina acknowledges support from the Claudia Adams Barr Program in Cancer Research. J.R. Brown acknowledges support from NIH grant K23 CA115682. T.M. Love is a National Research Service Award Fellow (T32CA70083).

Received for publication February 20, 2006, and accepted in revised form November 14, 2006.

Address correspondence to: Anthony Letai, Department of Medical Oncology, Dana-Farber Cancer Institute, 44 Binney Street, Dana 530, Boston, Massachusetts 02115, USA. Phone: (617) 632-2348; Fax: (617) 582-8160; E-mail: Anthony_Letai@dfci.harvard.edu.
1. Cory, S., and Adams, J.M. 2002. The Bcl2 family: regulators of the cellular life-or-death switch. Nat. Rev. Cancer. 2:647-656.

2. Danial, N.N., and Korsmeyer, S.J. 2004. Cell death: critical control points. Cell. 116:205-219.

3. Puthalakath, H., and Strasser, A. 2002. Keeping killers on a tight leash: transcriptional and posttranslational control of the pro-apoptotic activity of BH3-only proteins. Cell Death Differ. 9:505-512.

4. Kelekar, A., and Thompson, C.B. 1998. Bcl-2-family proteins: the role of the $\mathrm{BH} 3$ domain in apoptosis. Trends Cell Biol. 8:324-330.

5. Boyd, J.M., et al. 1995. Bik, a novel death-inducing protein shares a distinct sequence motif with Bcl-2 family proteins and interacts with viral and cellular survival-promoting proteins. Oncogene. 11:1921-1928.

6. Zong, W.X., Lindsten, T., Ross, A.J., MacGregor, G.R., and Thompson, C.B. 2001. BH3-only proteins that bind pro-survival Bcl-2 family members fail to induce apoptosis in the absence of Bax and Bak. Genes Dev. 15:1481-1486.

7. Wei, M.C., et al. 2001. Proapoptotic BAX and BAK: a requisite gateway to mitochondrial dysfunction and death. Science. 292:727-730.

8. Letai, A., et al. 2002. Distinct BH3 domains either sensitize or activate mitochondrial apoptosis, serving as prototype cancer therapeutics. Cancer Cell. 2:183-192.

9. Kuwana, T., et al. 2005. BH3 domains of BH3only proteins differentially regulate Bax-mediated mitochondrial membrane permeabilization both directly and indirectly. Mol. Cell. 17:525-535.

10. Green, D.R., and Kroemer, G. 2004. The pathophysiology of mitochondrial cell death. Science. 305:626-629.

11. Wang, X. 2001. The expanding role of mitochondria in apoptosis. Genes Dev. 15:2922-2933.

12. Cheng, E.H., Levine, B., Boise, L.H., Thompson, C.B., and Hardwick, J.M. 1996. Bax-independent inhibition of apoptosis by Bcl-XL. Nature. 379:554-556.

13. Cheng, E.H., et al. 2001. BCL-2, BCL-X(L) sequester $\mathrm{BH} 3$ domain-only molecules preventing BAX- and BAK-mediated mitochondrial apoptosis. Mol. Cell. 8:705-711.

14. Certo, M., et al. 2006. Mitochondria primed by death signals determine cellular addiction to antiapoptotic BCL-2 family members. Cancer Cell.

\section{9:351-365.}

15. Oltersdorf, T., et al. 2005. An inhibitor of Bcl-2 family proteins induces regression of solid tumors. Nature. 435:677-681.

16. Bouillet, P., et al. 1999. Proapoptotic Bcl-2 relative Bim required for certain apoptotic responses, leukocyte homeostasis, and to preclude autoimmunity. Science. 286:1735-1738.

17. Opferman, J.T., et al. 2003. Development and maintenance of $\mathrm{B}$ and $\mathrm{T}$ lymphocytes requires antiapoptotic MCL-1. Nature. 426:671-676.

18. Han, J., et al. 2004. Degradation of Mcl-1 by granzyme B: implications for Bim-mediated mitochondrial apoptotic events. J. Biol. Chem. 279:22020-22029.

19. Han, J., Goldstein, L.A., Gastman, B.R., and Rabinowich, H. 2006. Interrelated roles for Mcl-1 and Bim in regulation of TRAIL-mediated mitochondrial apoptosis. J. Biol. Chem. 281:10153-10163.

20. Kitada, S., et al. 1998. Expression of apoptosis-regulating proteins in chronic lymphocytic leukemia: correlations with in vitro and in vivo chemoresponses. Blood. 91:3379-3389.

21. Saxena, A., et al. 2004. Mcl-1 and Bcl-2/Bax ratio are associated with treatment response but not with Rai stage in B-cell chronic lymphocytic leukemia. Am. J. Hematol. 75:22-33.

22. Gomez-Bougie, P., Oliver, L., Le Gouill, S., Bataille, R., and Amiot, M. 2005. Melphalan-induced apoptosis in multiple myeloma cells is associated with a cleavage of Mcl-1 and Bim and a decrease in the Mcl-1/Bim complex. Oncogene. 24:8076-8079.

23. Wei, M.C., et al. 2000. tBID, a membrane-targeted death ligand, oligomerizes BAK to release cytochrome c. Genes Dev. 14:2060-2071.

24. Alves, N.L., et al. 2006. The Noxa/Mcl-1 axis regulates susceptibility to apoptosis under glucose limitation in dividing T cells. Immunity. 24:703-716.

25. Zhu, Y., et al. 2004. Constitutive association of the proapoptotic protein Bim with Bcl-2-related proteins on mitochondria in T cells. Proc. Natl. Acad. Sci. U. S. A. 101:7681-7686.

26. Marani, M., Tenev, T., Hancock, D., Downward, J., and Lemoine, N.R. 2002. Identification of novel isoforms of the $\mathrm{BH} 3$ domain protein $\mathrm{Bim}$ which directly activate Bax to trigger apoptosis. Mol. Cell. Biol. 22:3577-3589.

27. Yamaguchi, H., and Wang, H.G. 2002. Bcl-XL protects BimEL-induced Bax conformational change and cytochrome $\mathrm{C}$ release independent of interacting with Bax or BimEL. J. Biol. Chem. 277:41604-41612.

28. Chen, L., et al. 2005. Differential targeting of prosurvival Bcl-2 proteins by their BH3-only ligands allows complementary apoptotic function. Mol. Cell. 17:393-403.

29. Derenne, S., et al. 2002. Antisense strategy shows that $\mathrm{Mcl}-1$ rather than $\mathrm{Bcl}-2$ or $\mathrm{Bcl}-\mathrm{x}(\mathrm{L})$ is an essential survival protein of human myeloma cells. Blood. 100:194-199.

30. Green, D.R., and Evan, G.I. 2002. A matter of life and death. Cancer Cell. 1:19-30.

31. Hahn, W.C., and Weinberg, R.A. 2002. Modelling the molecular circuitry of cancer. Nat. Rev. Cancer. 2:331-341.

32. Calin, G.A., et al. 2005. A MicroRNA signature associated with prognosis and progression in chronic lymphocytic leukemia. N. Engl. J. Med. 353:1793-1801.

33. O'Brien, S.M., et al. 2005. Phase I to II multicenter study of oblimersen sodium, a Bcl-2 antisense oligonucleotide, in patients with advanced chronic lymphocytic leukemia. J. Clin. Oncol. 23:7697-7702.

34. Puthalakath, H., Huang, D.C., O’Reilly, L.A., King, S.M., and Strasser, A. 1999. The proapoptotic activity of the Bcl-2 family member Bim is regulated by interaction with the dynein motor complex. Mol. Cell. 3:287-296.

35. Letai, A., Beard, C., Sorcinelli, M., and Korsmeyer, S.J. 2004. Anti-apoptotic BCL-2 is required for maintenance of a model leukemia. Cancer Cell. 6:241-249.

36. Vaux, D.L., Cory, S., and Adams, J.M. 1988. Bcl-2 gene promotes haemopoietic cell survival and cooperates with c- myc to immortalize pre-B cells. Nature. 335:440-442.

37. McDonnell, T.J., et al. 1989. bcl-2-immunoglobulin transgenic mice demonstrate extended B cell survival and follicular lymphoproliferation. Cell. 57:79-88.

38. Dohner, H., et al. 2000. Genomic aberrations and survival in chronic lymphocytic leukemia. N. Engl. J. Med. 343:1910-1916.

39. Rassenti, L.Z., et al. 2004. ZAP-70 compared with immunoglobulin heavy-chain gene mutation status as a predictor of disease progression in chronic lymphocytic leukemia. N. Engl. J. Med. 351:893-901.

40. Ernst, P., Mabon, M., Davidson, A.J., Zon, L.I., and Korsmeyer, S.J. 2004. An Mll-dependent Hox program drives hematopoietic progenitor expansion. Curr. Biol. 14:2063-2069. 